- Nasal inhalation of cocaine ('snorting') can have severe destructive effects on the nasal and palatal tissues.

- The commonest causes of naso-palatal fistula are trauma, tumour, Wegener's granulomatosis and syphilitic gumma.

- Palatal defects can be repaired using a variety of techniques.

\title{
Palatal and nasal necrosis resulting from cocaine misuse
}

\author{
N. M. Goodger, ${ }^{1}$ J. Wang ${ }^{2}$ and M. A. Pogrel ${ }^{3}$
}

The potential social and economic harm caused by cocaine addiction is well known. The wider health risks and potential nasal complications of septal perforation and nasal collapse are also recognised. We report a case of oro-nasal fistula secondary to cocaine misuse and discuss the aetiology, management and potential methods of repair.

\section{INTRODUCTION}

Cocaine is an alkaloid, which was originally used by South American Indians as a stimulant. It was introduced to medicine by Köller in the 1880s as a local anaesthetic. More recently, its stimulant effects have led to its use as a recreational drug resulting in social, economic and physical harm. Oral intake of cocaine may result in anaesthesia of the tongue or gingiva. Inhalation of cocaine (snorting) has been associated with epistaxis, chronic rhinitis, nasal septal perforation and destruction of the lateral nasal walls which may lead to the saddle nose deformity. ${ }^{1}$ Reduction in the sense of smell, nasopharyngeal ulceration and dysphonia have also been described. However, the appearance of a large oro-nasal perforation in addition to destruction of the septum and lateral walls of the nose has only rarely been reported.

\footnotetext{
${ }^{1 *}$ Consultant Oral and Maxillofacial Surgeon, Kent and Canterbury Hospital, Ethelbert Road, Canterbury, Kent, CT1 3NG: ${ }^{2}$ Former resident, Department of Oral and Maxillofacial Surgery, University of California, San Francisco; ${ }^{3}$ Professor and Chairman, Department of Oral and Maxillofacial Surgery, University of California, San Francisco.

*Correspondence to: Dr Nicholas Goodger

Email: nicgoodger@aol.com
}

\section{Refereed Paper}

Received 21.04.04; Accepted 15.07.04

doi: 10.1038/sj.bdj.4812171

๑ British Dental Journal 2005; 198: 333-334

\section{CASE REPORT}

A 43-year-old Caucasian female presented with a $3 \mathrm{~cm}$ by $1.5 \mathrm{~cm}$ oro-nasal communication coupled with destruction of the nasal septum and lateral walls of the nose (Fig 1). She gave a five year history of nasal cocaine usage, initially at a frequency of two to three times a month but developing into a daily habit 18 months prior to presentation. The patient reported that she had always snorted through the left nostril.

Her initial symptoms were some thickening of the left nasal sill, and this was followed by a nasal septal perforation. The oro-nasal fistula developed as a small hole approximately three years prior to presentation and had gradually increased in size. She had chronic drainage from the communication and had been intermittently prescribed penicillin and clindamycin over the preceding six months. She denied cocaine use in the six months prior to referral but she still presented with multiple small bony sequestrae and areas of soft tissue necrosis. There was no history of trauma.

Under general anesthesia, the area was thoroughly debrided to include both hard and soft tissues, prior to planned secondary definitive reconstruction. Multiple specimens were sent for microbiology and histopathological examination. She healed well following this procedure, with no further areas of necrosis, and initially wore a removable obturator. Microbiology, histopathology and serological examination showed only necrotic bone and soft tissue with no other pathology. The patient was advised of the diagnosis and informed that further cocaine use would result in progression of the defect and prevent successful closure of the fistula. In order to monitor the compliance with this advice, the patient was followed up for six months after the initial debridement. At the end of this period the defect was observed to be static in size with healthy soft tissue margins and no evidence of necrosis or bony sequestrum. Definitive reconstruction was therefore undertaken using an anteriorly based lateral tongue flap to repair the defect after freshening of the wound edges. The patient was placed in intermaxillary fixation at the end of the procedure to safeguard the repair. The pedicle was divided under general anaesthetic three weeks later once the flap was vascularised from the periphery (Fig 2). The patient made a good post operative recovery but shortly thereafter was lost to follow up.

\section{DISCUSSION}

In addition to cocaine misuse, possible causes of midline palatal necrosis include the gumma of tertiary syphilis, tumour, trauma, chronic infection (often fungal), Wegener's granulomatosis, and midline granuloma, which is normally caused by 


\section{PRACTICE}

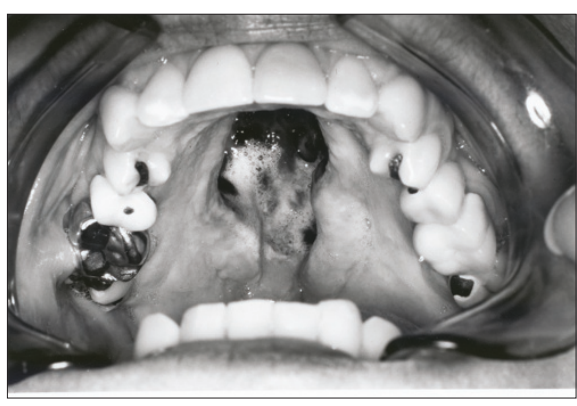

Fig. 1 Necrotic nasal tissue visible through midline palatal fistula

angiocentric T-cell lymphoma. The history, serology, microbiology and histopathology ruled these out as possible causes leaving only the cocaine misuse. The mechanism is thought to be the intense vasoconstriction caused by the cocaine, which causes necrosis of the affected tissues associated with chondritis and osteomyelitis of the exposed cartilage and bone. The thickening of the nasal sill prior to septal perforation in the current case was probably a manifestation of underlying chondritis.

Thirteen previous cases of palatal necrosis secondary to cocaine misuse are recorded in the literature. ${ }^{2-3}$ In only one of these cases - a 53-year-old man - was the destruction caused by granulomatous vasculitis confirmed by positive antineutrophil cytoplasmic antibody (ANCA) serology. It is of particular note that as in our case, 10 previous cases were in women with a mean age of 33.6 years. The relatively young age group may reflect the population to which cocaine is available rather than the disease process itself, since the remaining two male cases were 36 and 37 years old, but it has been suggested that females may be more susceptible to gross palatal necrosis in a similar way to the higher incidence of connective tissue disorders in women.

The most important tenet of treatment is cessation of the cocaine habit and the exclusion of another underlying disease process by biopsy and serology. Patients need to be counselled on the risks of continued cocaine misuse and the likely poor outcome from surgery if the misuse is continued. However, it can be difficult to check patient compliance. It is well recognised that those with a drug habit may be less than truthful about drug use and urine and blood tests will usually only detect metabolites from cocaine use 48-72 hours prior to the test. Thus, lack of deterioration of the palatal defect with no evidence of soft tissue necrosis or bone and cartilage sequestration may be a good indicator of a halt in cocaine use.

Treatment options include accepting the defect with or without permanent obturation $^{3}$ or surgical repair once a period of observation and non-progression of the defect has elapsed. Definitive surgical reconstruction could be by means of a cleft palate type procedure, a local pedicle flap such as the tongue flap used in this and other cases, ${ }^{3,4}$ temporalis flap or buccal fat pad graft. The pedicled tongue flap is a straightforward and reliable technique and provides good tissue bulk. Where extensive palatal destruction has occurred, a more versatile approach could be achieved using a microvascular free flap with or without simultaneous bone transfer. ${ }^{5,6}$ Suitable donor sites with bone would include the radial forearm, iliac crest, scapula and split metatarsal flap. Soft tissue reconstruction alone could be affected by the use of a radial forearm, dorsalis pedis or other musculocutaneous microvascular flap. The potential drawback to the use of microvascular reconstruction in this

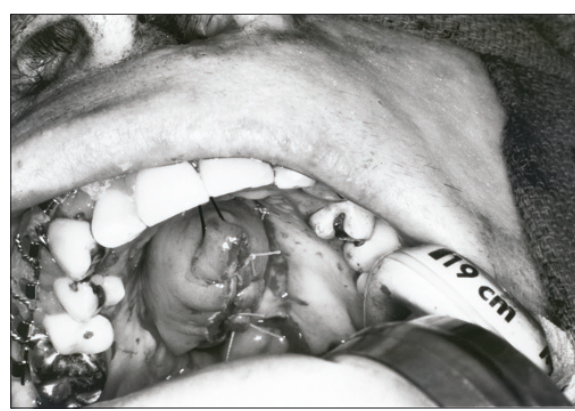

Fig. 2 Postoperative photograph showing palatal repair with tongue flap following division of the pedicle.

situation is that if the patient were to use cocaine in the postoperative period the resultant vasoconstriction could result in flap failure.

The loss of patients to follow up has been reported by others. ${ }^{3}$ This may be consistent with the patients' re-involvement with drugs. Patients are motivated to attend and receive treatment for the defect because of the nasal escape distorting speech and difficulty in eating, but once the treatment is complete that motivation may be lost.

1. Messinger E. Narcotic septal perforations due to drug addiction. J Am Med Assoc 1962; 179: 964.

2. Sittel $C$, Eckel $H$ E. Nasal cocaine abuse presenting as a central facial destructive granuloma. Eur Arch Otorhinolaryngol 1998; 255: 446.

3. Mari A, Arranz C, Gimeno X et al. Nasal cocaine abuse and centrofacial destructive process: report of three cases including treatment. Oral Surg Oral Med Oral Pathol Oral Radiol Endod 2002; 93 : 435-439.

4. Posnick J C, Getz S B. Surgical closure of end-stage palatal fistulas using anteriorly-based dorsal tongue flaps. J Oral Maxillofac Surg 1987; 45 907.

5. Turk A E, Chang J, Soroudi A E et al. Free flap closure in complex congenital and acquired defects of the palate. Ann Plast Surg 2000; 45: 274.

6. Futran N D, Haller J R. Considerations for free-flap reconstruction of the hard palate. Arch Otolaryngol Head Neck Surg 1999; 125: 665. 